\title{
New Trends in Non-Destructive Evaluation of Surface Hardened Layers and Coatings
}

\author{
Valery Vengrinovich
}

Laboratory of Computer Diagnostics, Institute of Applied Physics, National Academy of Sciences, Minsk, Belarus.

Email: veng@iaph.bas-net.by

Received November 29 $9^{\text {th }}, 2012$; revised March 10 $0^{\text {th }}, 2013$; accepted April $10^{\text {th }}, 2013$

Copyright (C) 2013 Valery Vengrinovich. This is an open access article distributed under the Creative Commons Attribution License, which permits unrestricted use, distribution, and reproduction in any medium, provided the original work is properly cited.

\begin{abstract}
This article should not be considered as a full review of current methods for non-destructive testing of surface layers. Rather, it is a subjective in this area. However, the article provides some review of the challenges posed by the current state of surface layers treatment techniques on the area of Non-Destructive materials evaluation: enhancement of the sensitivity to the type of defects, increasing resolution to submicron values, the requirement to diagnose the surface layers with depth resolution of properties, diagnosis of multilayer multicomponent surface layers and coatings, treated with concentrated energy.
\end{abstract}

Keywords: Surface Layers; Coatings; Properties; Non-Destructive Evaluation

\section{Introduction}

Due to the particular location, the surface layer of the material is in a specific state, and has properties that are often different from those of a base material. Common causes of this condition are well known:

- weakened condition of atomic bonds;

- interaction with the environment, characterized by oxidizing, hydrogen saturation, or other properties;

- defects of treatment appearance: mechanical, thermal, radiation, or other;

- segregation of impurities and defects, as in the case of fatigue or wear;

- the presence of tensile stress concentration zones, as in the case of grinding burns, etc.

Therefore, the surface material is always of particular concern to developers of products and designs. Structural and economic arguments add up to the need to provide at least equal strength and equivalence properties of the surface material and the substrate. This is achieved in two principal ways: a special coating or surface treatment. Application technology and processing have long turned to the independent industry, objecting to improve the operating characteristics of the articles by giving to their surface layers special properties like strength, resistance to wear, oxidation or fatigue, providing thermal stability, radiation and corrosion resistance, the ability to absorb or reflect light of certain wavelengths, etc. up to imparting to products the disinfecting or antibacterial features.

The important parts of surface treatment industry are diagnostic methods of surface layers and coatings, determination of their geometric, physical and chemical characteristics by a variety of NDT methods. It is clear that any one single method of treatment or coating is not complete without the development of technology and means for measuring or assessing the full range of properties of layers and/or coatings: thickness, hardness, stress, chemical composition and optical properties, thermal conductivity, surface roughness, fatigue strength and wear resistance, the presence of surface nonuniformity, etc. In now-a-day experts arsenal for surface layers diagnosis (SLD) there are instruments and methods of non-destructive testing: magnetic, eddy current, ultrasonic, radiation, thermal, magnetic noise, microwave, optical probe microscopy, etc. As I know, one of the first instruments for surface testing in mass production, was magnetic Akulov thickness meter (ATM) (Figure 1), based on the principle of the ponderomotive interaction of a permanent magnet with a ferromagnetic substrate. The instrument is able to measure within a few microns accuracy the thickness of non-magnetic materials (copper, tin, zinc, chromium, titanium) on steel, the thickness and properties of ferromagnetic nickel coatings on the same base material, the content of the ferromagnetic $\alpha$-phase in stainless steel, etc. The device had full metrological 


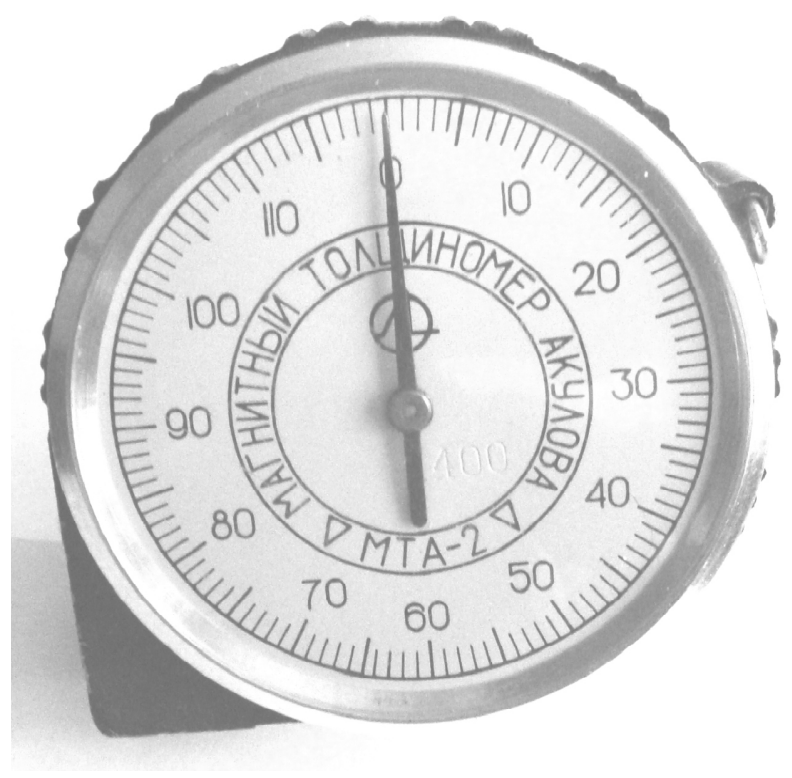

Figure 1. Akulov magnetic thickness gage.

support, including methods of certification, verification and measurement, and came with a set of calibration and control samples. Despite the fact that the device has been patented worldwide and put into production in 1963, still no one device in its class can compete with ATM in terms of accuracy, ease of use, reliability and portability.

Since that together with the emergence of a large number of methods for applying layers and surface treatment, the demands to SLD became more sophisticated. There is a need to measure the thickness of layers with the accuracy from nanometers (nano-layers) to a few millimeters (clad layers), the parameters of multilayer coatings, layers treated with concentrated (laser, plasma) sources of heat, cover the diagnosis of new materials (such as graphite), properties of coatings and layers with a depth resolution (for example, after surface plastic deformation (SPD)), to identify defects in nanoscale coatings and more.

The conventional market offers a large number of non-destructive and low-damaging devices for the diagnosis of layers and coatings: eddy current, ultrasonic, magnetic, microwave, micro-X-ray spectral and others. The set of measured parameters is usually reduced to the thickness, hardness and chemical composition. This article does not aim to describe all existing methods of traffic police. It attempts to present some, from the point of view of the author, significant trends in SLD and some nearest prospects of this development.

\subsection{Evaluation of Coatings and Layers with Depth Resolution. Multilayer Coatings}

Evaluation of coatings and layers with depth resolution or layer-by-layer ( $\mathrm{LbL}$ ) analysis of surface layers (SL) in the mathematical formulation is classified as significantly ill-posed inverse problem. For the first time in this form the problem was formulated in [1,2] for the LbL analysis of hardened layers on ferromagnetic materials by magnetic noise in the following form:

$$
v(y)=\int_{0}^{\infty} \phi(x, y, \sigma(x)) e^{-\alpha(y) x} \mathrm{~d} x,
$$

where $v(y)$-effective voltage of Barkhausen Noise (BN) in the overhead Barkhausen transducer (BT), depending on a varied during the experiment parameter $y$ (for example, magnetization current or $\mathrm{BN}$ frequency range); $\varphi(x)$-the contribution of the unit layer to the BN signal; $\sigma(x)$ - parameter under test e.g. stress value, dependent on the depth $x ; \alpha(x)$-the damping factor of the BN in ferromagnet, which generally depends on the parameter $y$. If the reconstructed function is the distribution of internal stresses in depth (after SPD), the task of stress value reconstruction with depth resolution can be expressed as the following integral equation:

$$
\tilde{v}(i)=\int_{0}^{f} \sigma(x) e^{-\alpha(i) x} \mathrm{~d} x
$$

where $f$ - the ultimate depth of the informative layer.

Equation (2) is ill-posed Fredholm equation of the 1st kind and can be solved by traditional Tikhonov regularization technique [3]. Based on this approach, the authors in the Institute of Applied Physics in Minsk developed hardware and software system INTROMAT for Industrial implementation of $\mathrm{LbL}$ on-line analysis of elastic stresses in the surface layers subjected to the SPD, or hardness after surface heat treatment like cementation. Figure 2 (solid line) shows the stress profiles obtained for samples of steel $300 \mathrm{M}$ after SPD by solving the Equation (2). For comparison, Figure 2 also shows the stress distribution on the same sample, but measured by an independent destructive method comprising of LbL by a known electro polishing technique. The figure shows that both methods are in good agreement. The error, which provides a reconstruction method does not exceed $20 \%$, which is sufficient for many practical applications.

The strong micro-magnetic $\mathrm{BN}$ characteristics dependence of internal stress or other SL properties is the physical basis of this technique. The situation is much more complex for nonmagnetic conductive materials, which conductivity is weakly dependent on stress. For most structural materials, these changes do not exceed $3 \%$ of the usual material conductivity. In this case, the use of the frequency spectrum change with stress variation is more reasonable. American researchers [4] developed technique for eddy current signals frequency spectra inversion for the reconstruction of the stress function 


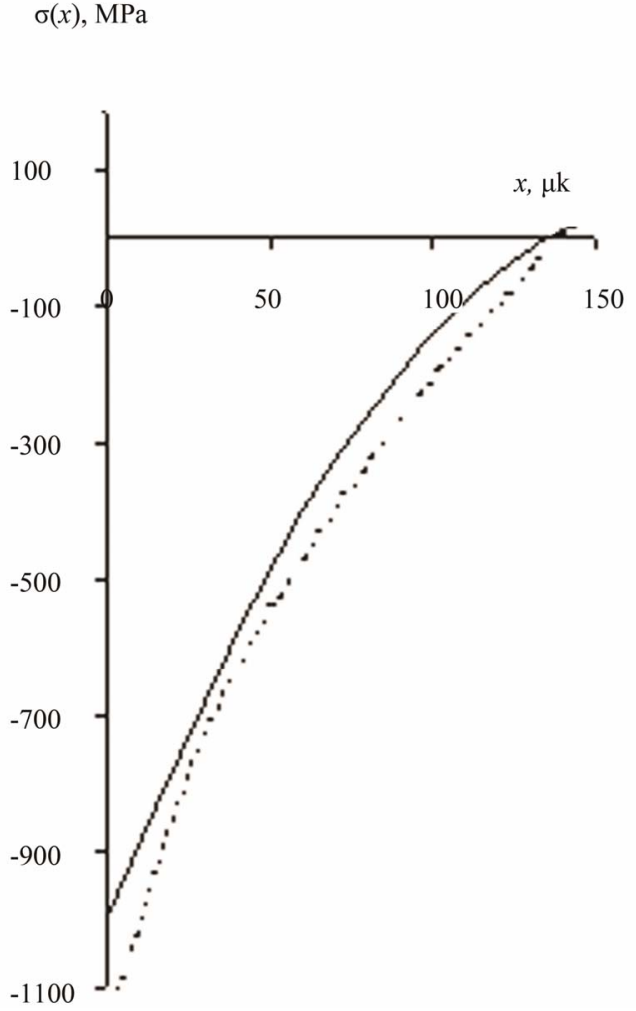

Figure 2. Layer-by-layer stress profiles $\sigma(x)$, acquired for steel 300M specimens by the solution of Equation (2) (dashed line) and measured by Davidenko method (electrolytic etching) (solid line) [2].

changes with depth resolution. The problem is central to space and aviation industry, where almost all the bearing components are subjected to surface hardening. Figure 3 shows an example of the imaginary part of the surface conductivity spectra variation (Apparent Eddy Current Conductivity, AECC) for samples of Inconel. As can be seen in a wide frequency range spectral characteristics vary by only $2.5 \%$. Therefore, a high-precision measurement of conductivity in the monitoring process is necessary. Figure 4 shows the results of the reconstruction of $\mathrm{LbL}$ function of stress distribution obtained by inverting AECC spectra with the simplified and iterative methods, respectively.

The need to solve the inverse problem (without restriction to a method of solution) for the reconstruction of LbL properties of SL was later independently confirmed and developed in many analysis. The use of methods of data inversion to study the properties of SL has been applied successfully, for example, to magnetic $[5,6]$, eddy current [7], and ultrasonic [8] methods. The list can be repeatedly extended. One of the most impressive application of inverse problems described in Several papers by G. Sabbah to reconstruct the multilayer coating properties of shuttles thermal protection [7] (Figure 5) by eddy current method. Left shows the compositions of layers,

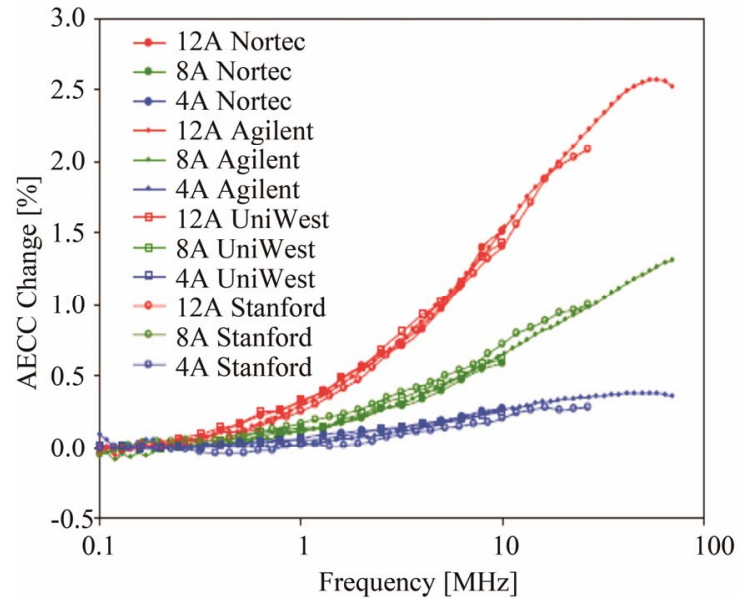

Figure 3. Apparent Eddy Current Conductivity (AECC) spectra in the INCONEL after surface plastic deformation treatment of the specimens with different Almen indexes, manufactured in different USA laboratories [4].

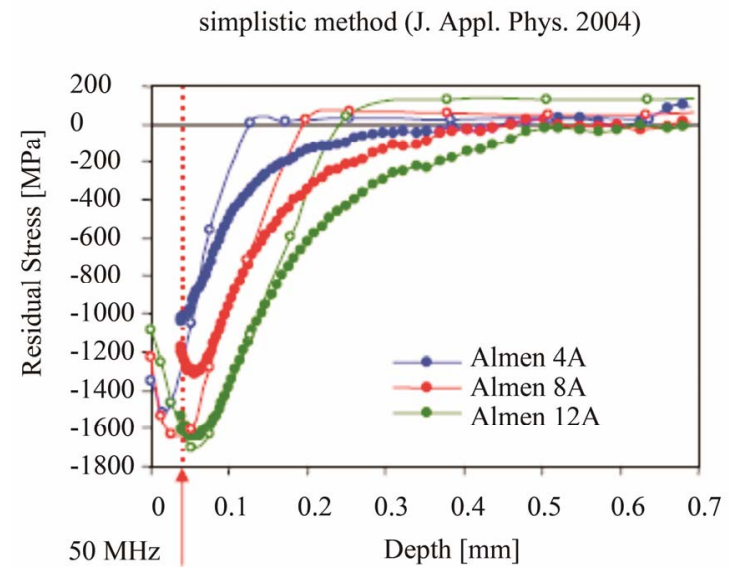

(a)

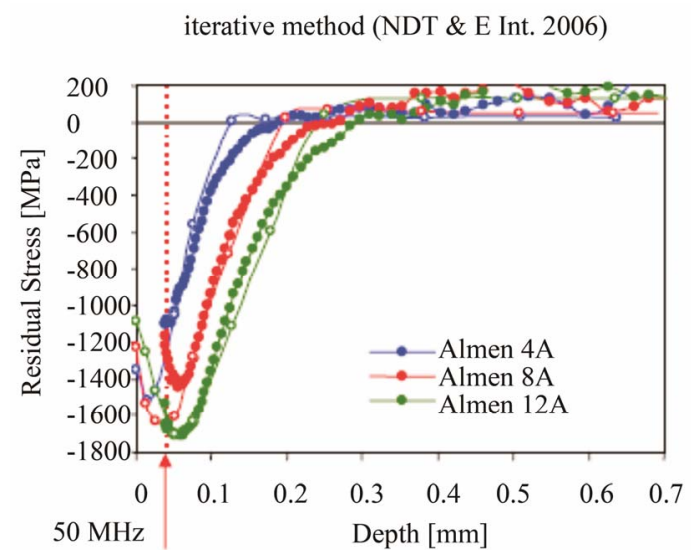

(b)

Figure 4. Reconstruction results of layer-by-layer stress distribution in the Inconel IN718 specimens with different Almen indexes, manufactured in different USA laboratories [4]. Reconstruction left-by simplified method, right-by iterative technique. Dotted lines-direct stress measurement results. 
and on the right shows the results of the reconstruction of their thickness. Stratified analysis is as well implemented in the framework of the thermal, X-ray and microwave methods.

\subsection{Magnetic Meander Magnetometers}

The meander Magnetic Winding Magnetometers $\left(\mathrm{MWM}^{\mathrm{TM}}\right)$ created first in Massachusetts Institute of Technology (MIT, USA) and then elaborated by Jentek Sensors Inc., are flexible film eddy current sensors for investigation the properties of conductive materials SL [9-11]. The uniqueness of the new technology is also in the use of so-called grid diagrams, actually the type of tetra-parameter hodograph chart, enabling to invert the measured amplitude and phase of the electromagnetic impedance values in to electric conductivity and magnetic permeability (Figure 6). Used up to temperatures $120^{\circ} \mathrm{C}$, MWM sensors are manufactured on a thin flexible substrate, making them adaptable to almost any surface. Intelligence surface depth depends on the excitation frequency and varies in the range from a part of a micron to a few parts of a millimeter. Sensors produced by planar technology, provide excellent reproducibility and resolution. MWM sensors and MWM-matrix are calibrated in air, they can be used as a high-precision measuring instruments of the absolute conductivity values. The equipment allows for a parallel interrogation of all the inputs, avoiding multiplexing and many times speeding up the measurement process as well as allowing the color imaging of the measured conductivity at large surfaces within a time close to real. Technology developerthe company JENTEK Sensors Inc., activated large number of applications to Non-Destructive Evaluation and diagnosis of the SL.
Figure 7 shows the distribution of the normalized permeability at the surface of the low-alloy steel plate in the process of tensile strain to failure. Images were obtained [12] by MWM matrix at $1 \mathrm{MHz}$ (a) and (b), and $158 \mathrm{kHz}$ - (c) and (d). Orientation of the coils with respect to the sample loading direction is shown on the right. Similar images have been obtained for electric conductivity distribution at different frequencies for other types of SL in conductive materials.

\subsection{Evaluation of Surface Layers Hardened with Concentrated Heat Sources}

The engineering industry orientation to promote the extended use of surface hardening technology, in particular, by concentrated heat sources (laser, electron beam and plasma) requires development of sensitive testing techniques. First the problem has been discussed in $[13,14]$, where the technology based on Barkhausen Noise was proposed. The efficiency of proposed technology later has been repeatedly confirmed. If one uses this, or any other instruments sensitive to SL properties, the most important questions remain locality and intelligence layer thickness, specific for the sensitivity of a transducer to the material structure and/or the internal stress, better with depth resolution. Also the selectivity with respect to other influencing factors is important. As shown in [15], $\mathrm{BN}$ technique mostly meets those requirements. The process of phase transformations at high heating rates (by laser heating they reach hundreds of thousands of degrees/sec) is very different from that occur during usual heating: critical transformation range (for phase recrystallization) is shifted toward higher temperatures as the square root of the speed heating. Therefore, even at temperatures close to the melting point, the usual hetero-

\begin{tabular}{|c|c|c|c|c|c|}
\hline$\beta$-phase depleted zone $2 \mathrm{Al}_{2} \mathrm{O}_{3}$ & $\begin{array}{c}\text { Sample } \\
\text { identification }\end{array}$ & $\begin{array}{l}\text { Coating } \\
\text { location }\end{array}$ & Coating identification & $\begin{array}{l}\text { Average } \\
\text { thickness } \\
\text { (microns) }\end{array}$ & $\begin{array}{l}\text { Computed } \\
\text { thickness } \\
\text { (microns) }\end{array}$ \\
\hline \multirow[t]{2}{*}{$\beta$-phase Aluminide } & \multirow{8}{*}{$\begin{array}{c}\text { 1950F-1000 } \\
\text { Cycles } \\
\text { (blue-4) }\end{array}$} & \multirow{4}{*}{ Top side } & Inner diffusion & 25.8 & 26.4 \\
\hline & & & Inner beta & 34.0 & 33.0 \\
\hline \multirow[t]{2}{*}{$\beta$-phase depleted zone 1} & & & Beta phase zone & 62.6 & 63.8 \\
\hline & & & Outer beta Inner diffusion & 37.5 & 41.5 \\
\hline Interdiffusion zone & & \multirow{4}{*}{$\begin{array}{c}\text { Bottom } \\
\text { side }\end{array}$} & Inner diffusion & 23.7 & 25.0 \\
\hline \multirow[b]{3}{*}{ GTD-111 Substrate } & & & Inner beta & 38.1 & 40.1 \\
\hline & & & Beta phase zone & 52.6 & 53.0 \\
\hline & & & Outer beta Inner diffusion & 33.2 & 34.8 \\
\hline
\end{tabular}

Figure 5. Left-composition of tetra-layer coating of the USA shuttle heat protection. Above-reconstruction results of four layers thickness and comparison with measured data [7]. 

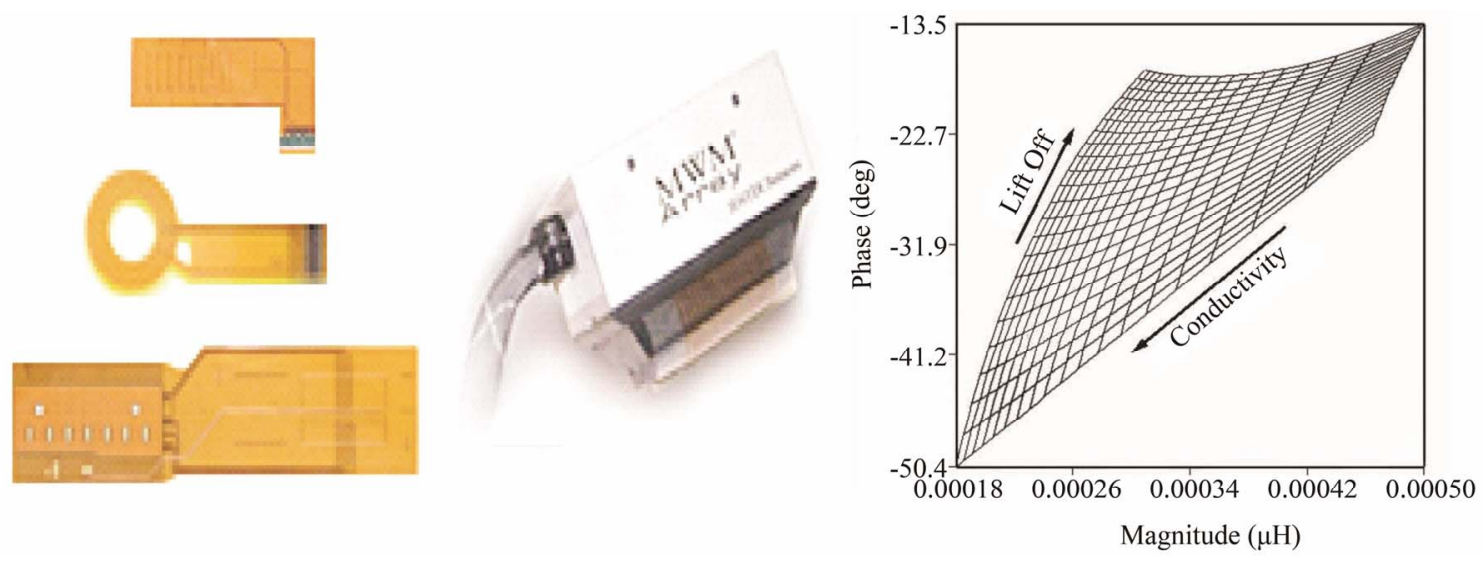

Figure 6. MWM sensors (left) and MWM array (center) by Jentek Sensors Inc. Right-tetra-parameter grid diagrams, allow to invert measured values of electromagnetic impedance into electric conductivity values [9-11].

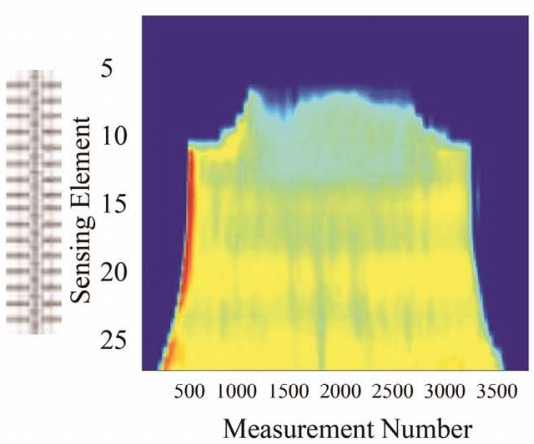

(a)

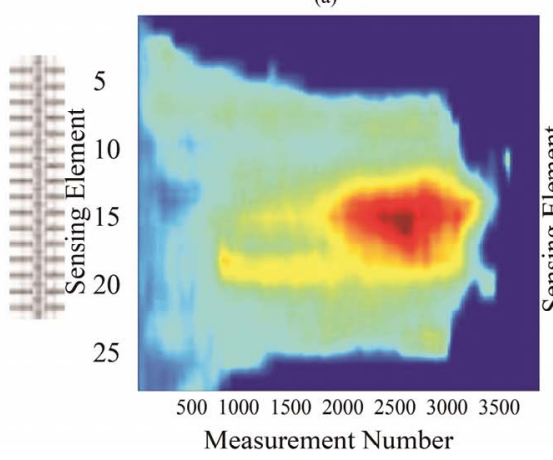

(b)
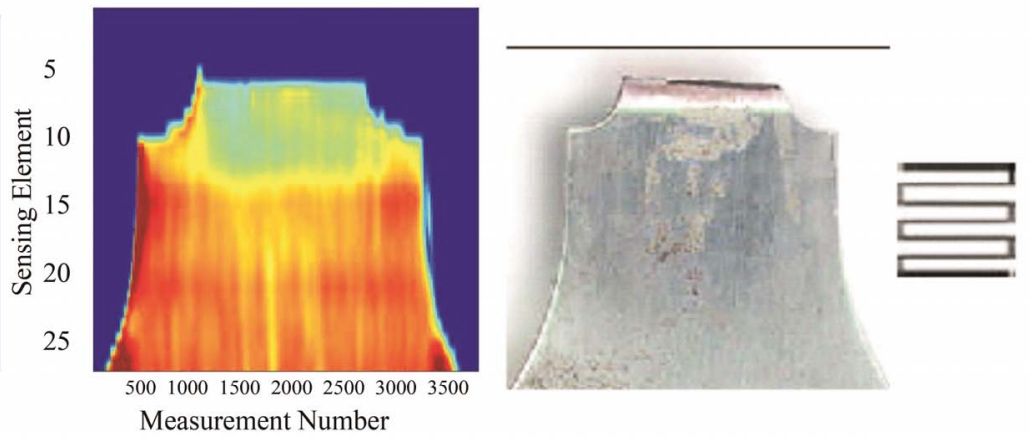

(c)
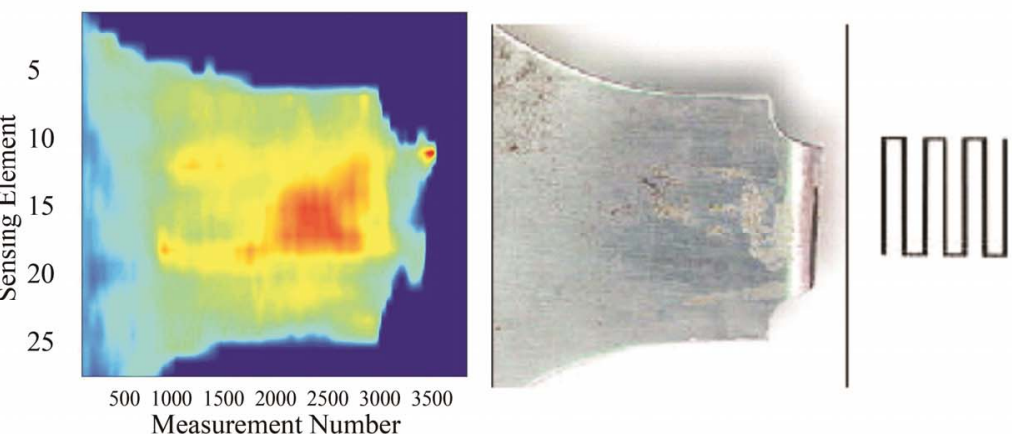

(d)

Figure 7. Normalized magnetic permeability distribution for one half of a double-notched, low-alloy 4340 steel specimen intra process its tensile deformation up to destruction. Images acquired by MWM-Array at: $1 \mathrm{MHz}$ (a) and (b), и $158 \mathrm{kHz}-$ (c) and (d). MWM array windings are shown on the left. Winding orientation with regard to the specimens is shown on the right [12].

geneous diffusion processes can not be realized, and, therefore, the structure of hardened layers and their thickness are determined not only by the front of the thermal conductivity. Therefore indirect Non-Destructive Evaluation (NDE) technique like infra-red method in this case is unacceptable. Strong difficulties arise in controlling the stability of quenching conditions (radiation power, scanning speed, the specific heat input, the diameter of the light spot, defocusing, edge effects), the degree of homogeneity of the original structure, the fluctuations of the absorption coefficient due to differences in surfaces conditions (corrosion, heat scale, surface contamination or damage). All this imposes specific requirements on the method of testing.

To improve the space resolution of the NDE method is necessary to reduce the diameter of the transducer. Figure 8 shows the results of the scan with Barkhausen transducer (profilograms) having the diameter $d=6.5$ $\mathrm{mm}$ across tracks of laser-hardened layers (LHL) in high carbon steel U10 with different ratios $l / d$, where $l=16$ 
$\mathrm{mm}$ - LUS track width (magnetization along LHL track). It can be seen that only the ratios $l / d>2.5$ are sufficient to determine the width of the track with available accuracy, while the actual depth can be reliably measured at $l / d>1$.

It should be mentioned the possibility of determining the bottom profile of LHL during scanning. Figure 8 on the right shows 5 profilograms of the relative value of magnetic noise intensity variation across the LHL track after consecutive electrolytic etching of the surface. The likelihood of bottom profile restoration is significantly increases with decreasing the thickness of the LHL.

Similar problems are also inherent in other treatments of a surface which give rise to surface damage, such as grinding burns or excessive wear, the NDE of which has to take into account other specific features of surface hardened layers. For example, the detection of grinding burns must provide a preliminary analysis of the nature of the burn, in particular, to assess whether the burn is of quenching or tempering type respectively. Otherwise, one can get results with the "back to front" accuracy. Preliminary assessment of the layer parameters measure- ing feasibility can be done using the formula, obtained in [2]:

$$
\gamma=1-S_{3} / S_{\text {д }}(1-h)\left(1-e^{-\alpha \delta}\right)
$$

where $\gamma=V / V_{\mathrm{H}}$ is the normalized value of the signal of magnetic noise, $V_{\mathrm{H}}=V_{\mathrm{H}}^{0} S_{\text {д }} / \beta$ the signal at $\delta \neq 0, \alpha$ and $\beta$-noise-damping coefficients in the hardened layer and the base metal, respectively, $V_{\mathrm{H}}^{0}$ - the value of

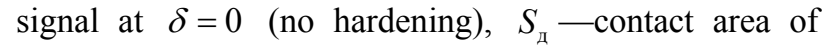
the sensor, $S_{3}$ - area of a hardened zone overlapped by a sensor, $h=V_{3} / V_{\mathrm{H}}$ - the ratio of the magnetic noise signal of hardened structure and basis material respectively.

\subsection{Some Approaches to NDE of Fatigue in Ferromagnetic Materials with Barkhausen Noise}

Metal fatigue always leads to a weakening of the surface. In the later stages of fatigue impurities and structure defects are segregated on the surface, generating successively slip lines, slip bands, surface steps, cavities and cracks. Thus, the role of the surface in the scenario of
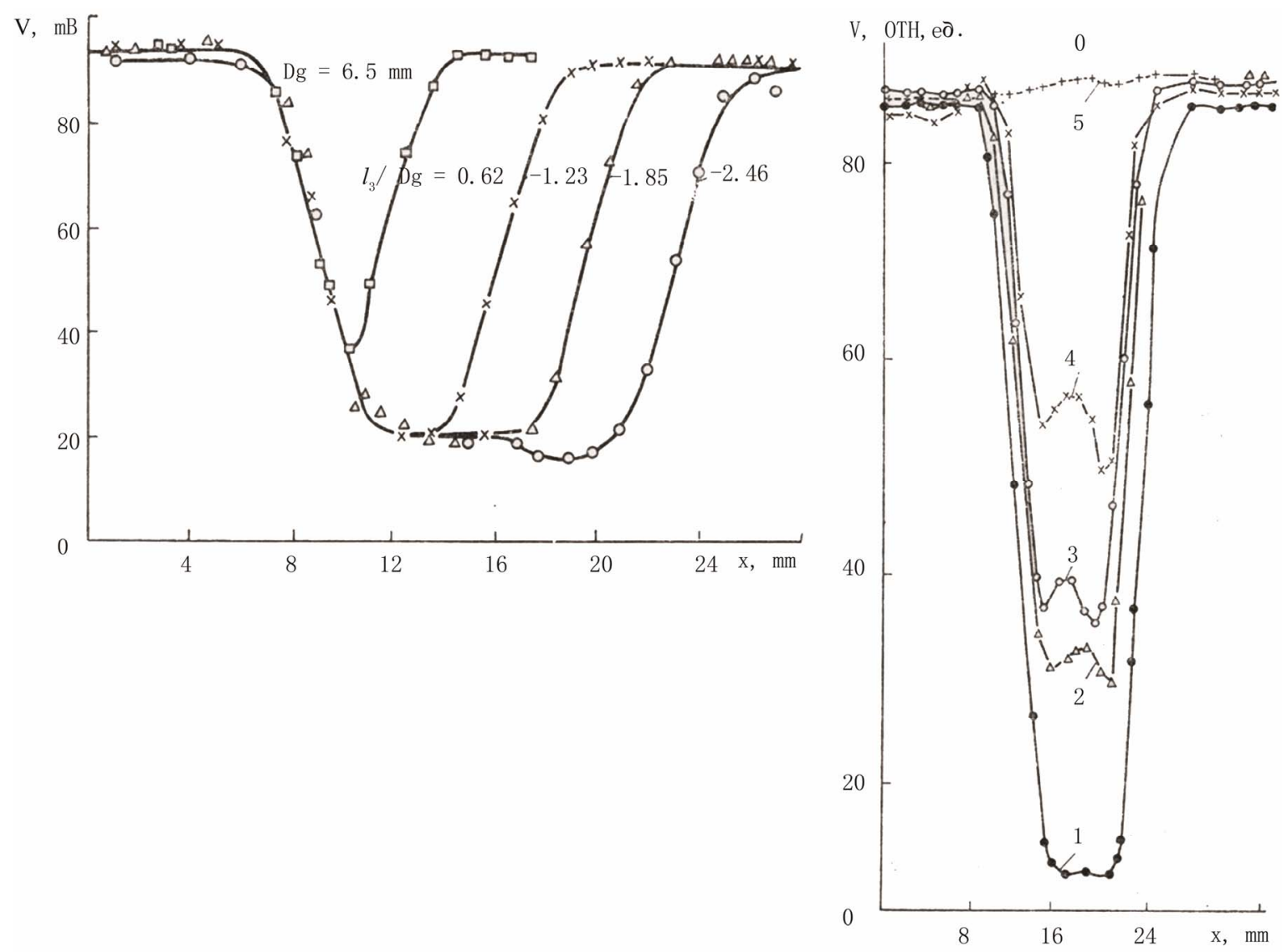

Figure 8. Above-Barkhausen noise probe $d=6.5 \mathrm{~mm}$ scanning results (profiles) across laser hardened trace in high carbon steel $\mathrm{U} 10$ at different ratio values $l / d$, where $l=16 \mathrm{~mm}$-trace width (magnetization-along laser trace). Right- 5 profiles across laser trace after consecutive electrolytic etching of the surface: 1) $\delta=750 \mu \mathrm{k}$; 2) $\delta=382 \mu \mathrm{k}$; 3) $\delta=312 \mu \mathrm{k}$; 4) $\delta=172 \mu \mathrm{k}$; 5) $\delta$ $=\mathbf{0} \boldsymbol{\mu k}$. 
fatigue degradation is significant. On the other hand, structural changes over fatigue process are not sufficient to cause significant changes in the micro structure characteristics, usually measured in non-destructive testing: the electrical conductivity, magnetic permeability, sound velocity, etc. Other material characteristics, like stress or grain microstructure, are much more influential. Therefore, until now a reliable diagnosis of fatigue in the early stages is very problematic. Significant progress was made in the study of fractal characteristics of the magnetic noise [16] in conjunction with the change of meso structure of steel. It is shown that the fractal dimension of Barkhausen Noise can quantitatively characterize the fatigue stage and the accumulation process of fatigue damage. This approach has already perfect hard- and software support. Another approach [17] is based on the measurements of the $\mathrm{LbL}$ distribution of $\mathrm{BN}$ parameters (Figure 9). LbL measurements of BN have been implemented after consecutive (step-by-step) removal of layers by electrolytic etching. The main conclusion is that, while the fatigue degradation of the metal is in progress, the $\mathrm{BN}$ properties of the layers at larger depths more and more differ from the BN properties on the surface. Thus, the progress in the NDE of fatigue can be achieved if one can measure the difference between BN power on the surface and in the subsurface layer respectively. The LbL technique described above is gives one of the problem solution.

\section{A New Look at Penetrant Inspection}

Penetrant testing $(\mathrm{PT})$ - the traditional method of detecting defects in the surface layers. Several questions were always critical: sufficiency of a contrast while detecting very small defects, problems of calibration and metrol- ogy and the lack of productivity. Although the daily practice of PT has changed little in recent years, the scientific progress achieved considerable success. The last is based on the achievements of the theory of hydrodynamic processes underlie penetrant inspection methods $[18,19]$. Shortly it can be reduced to the elaborations as follows:

- the new phenomena of bilateral filling of the deadend capillary [20], what means that the liquid fills the capillary not only from its open side, but also from the dead-end, the filling of the last being started usually before the open side part. This phenomena influences the sensitivity of PT. The mechanism of bilateral filling effect is realized by the flow of the polar liquid along the capillary walls from the external meniscus to the internal one;

- the soft- and hardware for detection, recognition and quantitative analysis of the images of indicator trace during PT;

- increasing sensitivity of PT by means of penetrant and developer heat treatment during application. For example, temperature growth by $20^{\circ} \mathrm{C}-40^{\circ} \mathrm{C}$ reduces the sensitivity threshold approximately twice and the penetration time - more than by one order;

- positive prospects has also the electrochemical treatment of the surface before testing, which "opens" the defect's edges and increases the PT sensitivity;

- the penetrant polarity is strongly affects PT productivity: it delays the flow of the penetrant during bilateral filling more than twice. The suggested values of penetrant's dielectric permeability $\varepsilon$ are optimal at $17<\varepsilon<25$.

The recommended results make it possible to increase the PT efficiency growth substantially.

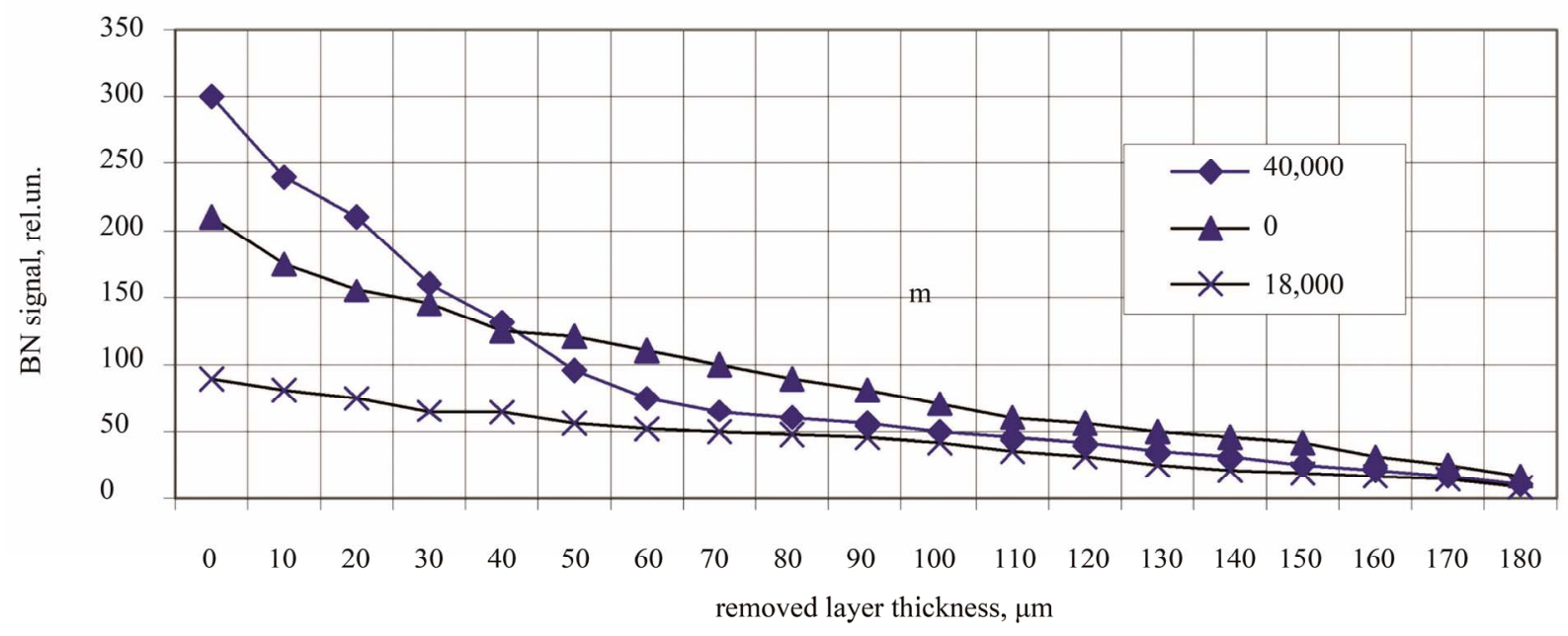

Figure 9. Barkhausen noise signal profile dependence via the thickness of the layer, removed by electrolytic etching, after different steps of martensite-aging alloy VNS-2 plate cycling: before cycling, after 18,000 cycles and after 40,000 cycles (crack appearance) [17]. 

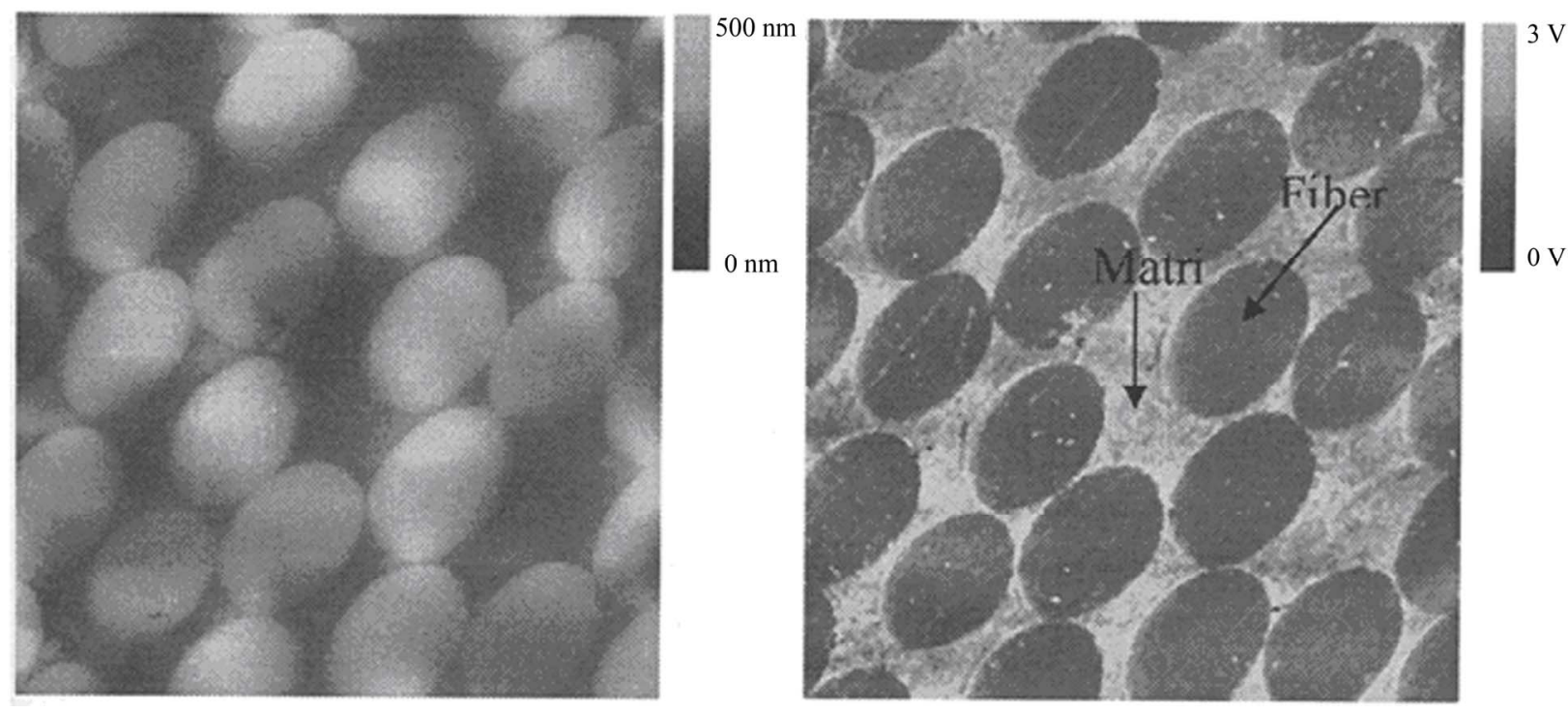

Figure 10. Topographic visualization of reinforced carbon fibers on the polymer matrix surface: left-with the help of usual atomic force microscope (AFM), right-eddy current AFM [19].

\section{NDE of Nanoscale Surface Defects}

This article does not address the numerous methods for the evaluation of surface nanostructures: atomic force, optical and X-ray electron microscopy. All those methods are more or less destructive, having in mind necessary precise surface treatment before testing. However, the sensitivity and thus requirements to surface treatment could be reduced by combining them with classical NDE techniques. The proposed one is eddy current imaging using an atomic force microscope (AFM). [21]. Usually the spatial resolution of eddy current flaw detectors is limited by the values 500 microns. However, the combination of eddy current NDE and AFM imaging of local changes in the electrical conductivity on the surface of products can achieve sub-micron resolution. Figure 10 shows the topographic images of carbon fiber reinforcement in a polymer matrix, obtained: left-with a conventional atomic force microscope, right-eddy current AFM. High resolution of a few nanometers is achieved by applying a magnetic film on a regular AFM tip of silicon nitride $10 \mathrm{~nm}$ in diameter, which is pressed with a force of $0.1 \mathrm{~N} / \mathrm{m}$. The product is mounted on the coil, excited by an AC power source with a frequency of $50-500 \mathrm{kHz}$. The magnetic field, generated by the coil, causes the oscillating magnetic tip and, therefore, the AFM cantilever, whose movement is recorded by the measurement device. The combination of traditional NDE methods and new systems for the study of submicron structures can be very promising for the study of new materials and methods for surface hardening.

\section{Conclusion}

This article should not be considered as a full review of current methods of non-destructive testing of surface layers. Rather, it is a subjective perspective on the trends in this area. However, the article provides some review of the challenges posed by the current state of surface layers treatment techniques on the area of Non-Destructive materials evaluation: enhancement of the sensitivity to the type of defects, increasing resolution to submicron values, the requirement to diagnose the surface layers with depth resolution of properties, diagnosis of multilayer multicomponent surface layers and coatings, treated with concentrated energy.

\section{REFERENCES}

[1] V. L. Vengrinovich, M. A. Knyazev and A. L. Vishnevsky, "The Principles of Depth Analysis of Surface Hardened Layers by Magnetic Noise Method," Research in Nondestructive Evaluation, Vol. 4, No. 1, 1992, pp. 1927.

[2] V. L. Vengrinovich, "Barkhausen Effect: Theory and Application,” Nauka I Technika, Minsk, 1991. (in Russian)

[3] V. Ya. Arsenin, "Methods of Mathematical Physics and Special Functions," 2nd Edition, Nauka, Moscow, 1984. (in Russian)

[4] Bassam A. Abu-Nabah, Feng Yu and Peter B. Nagy, "Electromagnetic Techniques for Residual Stress Measurements," University of Cincinnati, Cincinnati, Lecture Made in 1 Workshop on Residual Stress Measurements, Colorado, 2006.

[5] A. C. Bruno, "Imaging Flaws in Magnetically Permeable Structures Using the Truncated Generalized Inverse on Leakage Fields," Journal of Applied Physics, Vol. 82, No. 12, 1997, pp. 5899-5906. doi:10.1063/1.366490

[6] V. L. Vengrinovich, S. A. Emelyanenkov and G. R. Tillack, "2D Flaw Image Restoration from Magnetic Leak- 
age Field Data Using Singular Value Decomposition and Bayesian Techniques," 9th International Workshop on Electromagnetic Nondestructive Evaluation (ENDE'2003), Saclay, 15-16 May 2003, pp. 25-31.

[7] H. A. Sabbach, E. H. Sabbach, R. K. Murthy and J. Nyenhuis, "Assessing Thermal Barrier Coatings by Eddy Current Inversion," In: D. Thompson and D. Chimenti, Eds., Review of Progress in QNDE, Vol. 21, American Institute of Physics, 2002, pp. 722-727.

[8] A. R. Baev, A. L. Mayorov and M. A. Tischenko, "Ultrasonic Method for the Analysis of Metallic Materials Surface Hardening," Journal of Casting and Metallurgy, No. 4, 2010, pp. 167-271. (in Russian)

[9] N. J. Goldfine, et al., "Scanning and Permanently Mounted Conformable MWM Eddy Current Arrays for Fatigue/Corrosion Imaging and Fatigue Monitoring," USAF ASIP Conference, San Antonio, TX, 6 December 2000.

[10] N. J. Goldfine, et al., "Surface Mounted Periodic Field Eddy Current Sensors for Structural Health Monitoring," SPIE Conference: Smart Structures and Materials, NDE for Health Monitoring and Diagnosis, Newport Beach, 4-8 March 2001.

[11] V. Zilberstein, D. Schlicker, K. Walrath, V. Weiss and N. Goldfine, "MWM Eddy Current Sensors for Monitoring of Crack Initiation and Growth during Fatigue Tests and in Service," International Journal of Fatigue, Vol. 23, Supplement 1, 2001, pp. S477-S485. doi:10.1016/S0142-1123(01)00154-2

[12] V. Zilberstein, M. Fisher, D. Grundy, D. Schlicker, V. Tsukernik, V. Vengrinovich and N. Goldfine, "Residual and Applied Stress Estimation from Directional Magnetic Permeability Measurements with MWM Sensors," Journal of Pressure Vessel Technology, Vol. 124, No. 3, 2002, pp. 375-381.

[13] V. L. Vengrinovich, S. A. Astapchik and V. B. Babushkin, "Pecularities of the Structure Zones Evaluation after Lazer-Beam Hardening," Journal of Physics and Chemistry of Material Treatment, No. 6, 1984, pp. 10-15. (in
Russian)

[14] V. L. Vengrinovich, V. P. Yakunin, S. D. Legotin and V. N. Busko "Parameters Testing of Laser-Beam Layers in Ferromagnetic Materials by Barkhausen Noise Method," Russian Journal of NDT, No. 2, 1986, pp. 87-89. (in Russian)

[15] S.D. Legotin, "Parameters Testing of Laser-Beam Layers in Ferromagnetic Materials by Barkhausen Noise Method," Doctor Thesis, Institute of Applied Physics, Minsk, 1989. (in Russian)

[16] J. Screiber, U. Cikalova and Ye. Vertyagina, "Use of the Fractal Nature of Spatial and Temporal Response Behavior for Materials Damage Characterization," In: P. D. Portella, T. Beck and M. Okazaki, Eds., Proceedings of the 6th International Conference on Low Cycle Fatigue, Berlin, 2007, pp. 655-660.

[17] V. L. Vengrinovich and V. N. Busco, "New Approach to Fatigue Damage Evaluation via Barkhausen Noise," 10th European Conference on NDT, Moscow, June 2010, pp. 1-6.

[18] P. P. Prochorenko and N.N. Migoun, "Introduction to the Theory of Penetrant Testing," Science and Technology, Minsk, 1988. (in Russian)

[19] N. P. Migoun and A. I. Shnipp, "The Model of Film Liquid Flow in a Conical Dead-End Capillary," Journal of Engineering Physics, Vol. 75. No. 64, 2002, pp. 145-150. (in Russian)

[20] G. I. Dovgyallo, N. P. Migoun and P. P. Prochorenko, "On a Complete Filling of a Conical Dead-End Capillaries with Liquids," Journal of Engineering Physics, Vol. 56, No. 4, 1989, pp. 563-565. (in Russian)

[21] V. Nalladega, S. Sathish, K. V. Yata and M. P. Blodgett, "High Resolution Eddy Current Imaging with Atomic Force Microscope," In: D. Thompson and D. Chimenti, Eds., Quantitative Nondestructive Evaluation, American Institute of Physics, New York, 2008, pp. 400-405. 